\title{
Localización de fracturas en el mieloma múltiple en el Hospital Regional "Gral. Ignacio Zaragoza” ISSSTE
}

\author{
Incidence of the location of fractures in the multiple myeloma in \\ the Regional Hospital "Gral. Ignacio Zaragoza" ISSSTE
}

\author{
Angélica Maricela Carrillo Sánchez, ${ }^{*}$ Luis Alberto De Santiago Esquivel,* Claudia Karina Pérez García, \\ Carlos Eduardo Antonio Romero, ${ }^{\S}$ Juan Manuel Pérez Zúñiga, " Denisse Añorve Bailon॥ \\ Citar como: Carrillo SAM, De Santiago ELA, Pérez GCK, Antonio RCE, Pérez ZJM, Añorve BD. \\ Localización de fracturas en el mieloma múltiple en el Hospital Regional "Gral. Ignacio Zaragoza" \\ ISSSTE. Acta Med GA. 2021; 19 (4): 497-500. https://dx.doi.org/10.35366/102534
}

\section{Resumen}

El mieloma múltiple es una neoplasia hematológica derivada de alteraciones en las células de estirpe linfoide $\mathrm{B}$, se caracteriza por anomalías esqueléticas denominadas enfermedad ósea del mieloma múltiple. Objetivo: Determinar la localización de las fracturas en pacientes con mieloma múltiple del Hospital Regional "Gral. Ignacio Zaragoza" ISSSTE de 2016 a 2019. Material y métodos: Estudio retrospectivo; revisamos los expedientes clínicos radiológicos de pacientes diagnosticados con mieloma múltiple en el periodo de enero de 2016 a diciembre de 2019. Resultados: Incluimos 33 pacientes con mieloma múltiple. Tres pacientes (9.1\%) presentaron fractura con características de proceso neoplásico y posterior diagnóstico de mieloma múltiple. Otros pacientes presentaban síntomas de enfermedad ósea del mieloma múltiple: lumbalgia en $42.4 \%(n=14)$, dolor óseo generalizado en 15.2\% ( $n=5)$. En 24 pacientes documentamos fractura asociada al mieloma múltiple: columna en $39.4 \%(n=13)$, húmero en $12.1 \%(n=4)$, cadera en $9.1 \%$ $(n=3)$, esqueleto apendicular en $9.1 \%(n=3)$ y fémur en $3 \%$ $(n=1)$. Conclusión: Se diagnosticaron 24 pacientes $(78.7 \%)$ con fractura asociada al mieloma múltiple, los más frecuentes fueron en columna, húmero y cadera.

Palabras clave: Mieloma múltiple, enfermedad ósea del mieloma múltiple, fractura.

\section{Abstract}

Multiple myeloma is a hematological neoplasm derived from alterations in B lymphoid cells characterized by skeletal abnormalities called multiple myeloma bone disease. Objective: Determine the location of fractures in patients with multiple myeloma at the Regional Hospital "Gral. Ignacio Zaragoza" from 2016 to 2019. Material and methods: Retrospective study, we reviewed the radiological clinical records of patients diagnosed with multiple myeloma in the period from January 2016 to December 2019. Results: We included 33 patients with multiple myeloma. Three patients (9.1\%) presented a fracture with characteristics of a neoplastic process and subsequent diagnosis of multiple myeloma. Other patients had symptoms of multiple myeloma bone disease: low back pain $42.4 \%(n=14)$, generalized bone pain $15.2 \%$ $(n=5)$. In 24 patients, we documented a fracture associated with multiple myeloma: spine $39.4 \%(n=13)$, humerus $12.1 \%$ $(n=4)$, hip 9.1\% $(n=3)$, appendicular skeleton $9.1 \%(n=3)$ and femur $3 \%(n=1)$. Conclusion: 24 patients $(78.7 \%)$ were diagnosed with a fracture associated with multiple myeloma, being more frequent in the spine, humerus, and hip.

Keywords: Multiple myeloma, multiple myeloma bone disease, fracture.
* Médico Residente de cuarto año de Traumatología y Ortopedia, Hospital Regional "Gral. Ignacio Zaragoza". Alumno de la Facultad Mexicana de Medicina, Universidad La Salle. México.

₹ Médico Residente de quinto año de Cirugía Articular, Centro Médico Nacional 20 de Noviembre, UNAM. México.

$\S$ Profesor Titular del curso de Traumatología y Ortopedia, Hospital Regional "Gral. Ignacio Zaragoza". México.

" Médico adscrito al Servicio de Hematología, Hospital Regional "Gral. Ignacio Zaragoza". México.
" Médico adscrito al Servicio de Oncología Médica y encargada del Departamento de Enseñanza e Investigación, Hospital Regional "Gral. Ignacio Zaragoza". México.

Correspondencia:

Angélica Maricela Carrillo Sánchez

Correo electrónico: angelicam.carrillos@gmail.com

Aceptado: 19-01-2021.

www.medigraphic.com/actamedica

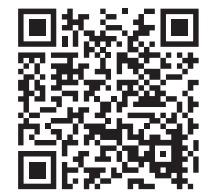




\section{INTRODUCCIÓN}

El mieloma múltiple (MM) es una neoplasia hematológica derivada de alteraciones en las células de estirpe linfoide B en su último estadio madurativo, por lo que son capaces de producir una paraproteína monoclonal que se asocia al desarrollo de características clínicas como anemia, insuficiencia renal, hipercalcemia o lesiones óseas. ${ }^{1}$ Es el segundo tipo de cáncer hematológico más frecuente. ${ }^{1}$ Tiene una incidencia anual de 22,000 casos a nivel mundial, ${ }^{2}$ la cual se incrementa con la edad, siendo más común en el sexo masculino y en ascendencia afroamericana. ${ }^{3}$

En México el mieloma múltiple corresponde de 4-8\% de los padecimientos hematooncológicos. ${ }^{4}$ La tasa de supervivencia es de $46.6 \%$ a cinco años. ${ }^{5}$ La etiología es desconocida, aunque se han descrito múltiples factores de riesgo en los que se incluyen condiciones genéticas (ciclinas, gen IgH o trisomías), epigenéticas (asociadas con la metilación) y cambios en la evolución clonal. ${ }^{6}$

En el MM se incrementa la actividad de los osteoclastos y disminuye el número de los osteoblastos conduciendo a osteoporosis y por consiguiente al desarrollo de lesiones líticas en el hueso. Esto se lleva a cabo por la interacción de las células plasmáticas malignas y las células del microambiente de la médula ósea. ${ }^{7}$

Todo este proceso conlleva un aumento en las células del mieloma múltiple, creando un círculo vicioso entre la expansión tumoral y la destrucción ósea, ocasionando lo que se conoce como enfermedad ósea del mieloma múltiple (EOMM). ${ }^{8}$ Ésta puede ser dolorosa y presentar lesiones líticas que incrementan el riesgo de fracturas hasta en nueve veces comparado con la población sin dicha enfermedad, deteriorando la calidad de vida de los pacientes. ${ }^{9,10}$

Las fracturas en terreno patológico que se encuentran en el MM pueden desarrollarse en la mayoría de los huesos; sin embargo, se encuentran más frecuentemente en las vértebras y en los huesos largos como el húmero, el fémur o las costillas. La localización, sin duda, impacta en las comorbilidades que genera. ${ }^{10}$

La distribución de las fracturas puede deberse a la infiltración de las células plasmáticas en el hueso trabecular similar al de la columna en comparación con el hueso menos hematopoyético como el cortical, lo que da como resultado la predilección de estas fracturas en regiones con mayor cantidad de trabéculas en su estructura (Figuras 1 y 2). ${ }^{11}$

El objetivo es determinar la localización de las fracturas en pacientes con diagnóstico de MM, según criterios establecidos por el Grupo Internacional de Trabajo del Mieloma (IMWG, por sus siglas en inglés) ${ }^{12}$ en el año 2014, en pacientes atendidos en el Hospital Regional "Gral. Ignacio Zaragoza" del ISSSTE.

\section{MATERIAL Y MÉTODOS}

Se realizó un estudio retrospectivo en el cual se revisaron los expedientes de pacientes diagnosticados con MM en el periodo de enero de 2016 a diciembre de 2019 . Asimismo, revisamos el archivo radiológico de estos pacientes. Los datos obtenidos se registraron en una hoja de recolección de datos por paciente y se concentraron en una hoja de Excel, realizando estadística descriptiva en el programa SPSS versión 20. Los gráficos fueron realizados en el programa GraphPad Prism versión 7. El estudio fue registrado y autorizado en el comité local de investigación.

\section{RESULTADOS}

De enero de 2016 a diciembre de 2019 se diagnosticaron en nuestra unidad 33 pacientes, según criterios del IMWG. El $57.5 \%$ de los pacientes fueron mujeres, la mediana de edad fue de 52 años, el estado funcional medido por la escala ECOG se encontró de 0 a 2 (97\%). El tipo de mieloma más frecuente fue la inmunoglobulina G (IgG) (66.7\%). Las características completas de los pacientes incluidos se describen en la Tabla 1.

Al analizar los síntomas de la enfermedad ósea al diagnóstico, se documentó que 9.1\% $(n=3)$ de los pacientes tuvieron una fractura que presentaba características radiológicas de proceso neoplásico con sospecha y posterior diagnóstico confirmatorio de MM. Sin embargo, no todos los pacientes presentaban una fractura que sugiriera $M M$, algunos pacientes al momento del diagnóstico compartieron características clínicas de la EOMM, en las cuales encontramos: lumbalgia en $42.4 \%$ $(n=14)$, dolor óseo generalizado en $15.2 \%(n=5)$, y $33.33 \%$ de los pacientes tenían un curso asintomático de la enfermedad (Figura 1).

Durante el seguimiento, se diagnosticaron 24 pacientes con alguna fractura asociada al mieloma múltiple, la localización de éstas se encontró con la siguiente distribución: columna en $39.4 \%(n=13)$, húmero en $12.1 \%(n=4)$, cadera en $9.1 \%(n=3)$, fracturas con otra localización fuera del esqueleto axial en $9.1 \%(n=3)$ y fémur en $3 \%(n=$ 1). Por otro lado, $27.3 \%$ de los pacientes no presentaron fracturas en el tiempo de seguimiento de este estudio.

Se realizó análisis de estudios de gabinete para encontrar datos radiográficos de la EOMM. De éstos, se excluyeron los hallazgos con trazo evidente de fractura, ya que varias características encontradas favorecerán la aparición de éstas, además de que denotan un estadio avanzado de la enfermedad. Al revisar los datos radiológicos de la enfermedad ósea encontramos que 30.3\% ( $n=10)$ de los pacientes presentaban lesiones líticas a nivel de la columna, 

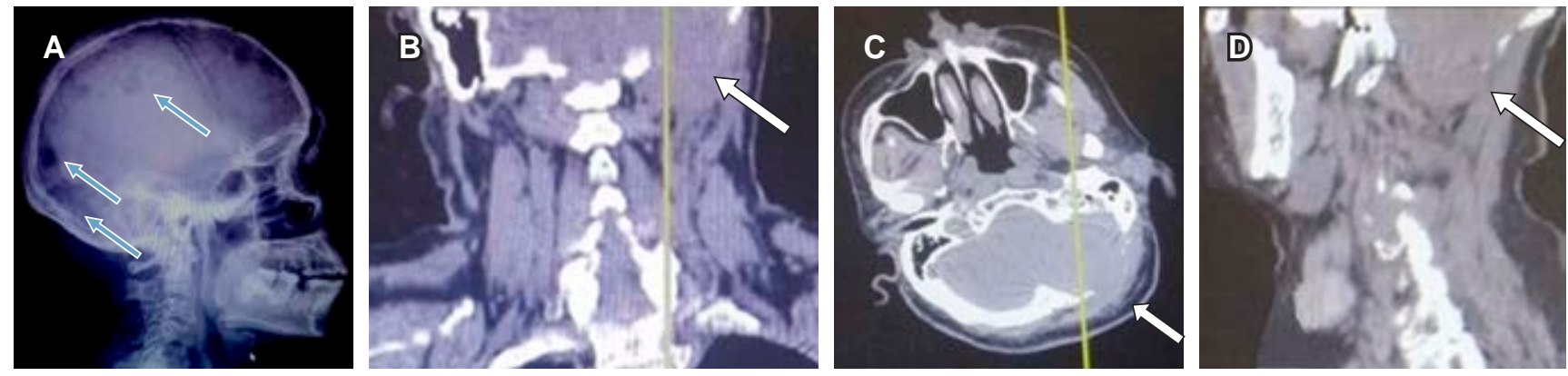

Figura 1: A) Radiografía de cráneo con lesiones líticas clásicas de mieloma múltiple (en sacabocado, flechas azules). B-D) Las flechas blancas representan un plasmocitoma en cráneo, en diferentes planos de corte de la tomografía de cráneo.

Figura 2:

RMN corte sagital de columna lumbar con lesiones líticas multinivel y fractura a nivel de L1.

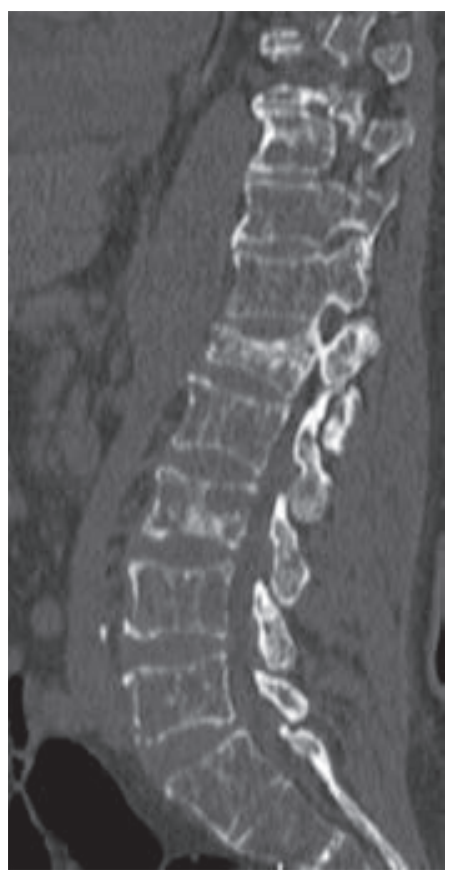

la presencia de plasmocitoma se encontró en 18.8\% (n $=8)$, lesiones líticas multinivel en $18.18 \%(n=7)$-estas lesiones incluyen más de un nivel vertebral, así como la inclusión de sacro o huesos largos-, destrucción vertebral en $6.1 \%(n=2)$ y en $21.2 \%(n=7)$ no se encontraron datos de enfermedad ósea del mieloma múltiple durante el seguimiento de los mismos.

\section{DISCUSIÓN}

Una de las manifestaciones del MM es el compromiso a nivel óseo que ocasiona, el cual puede presentarse de 70 a $80 \%$ de los pacientes ${ }^{13,14}$ y son estas manifestaciones las que incrementan el riesgo de eventos relacionados al esqueleto (ERE) que incluye a las fracturas en terreno
Tabla 1: Características de los pacientes $(\mathrm{N}=33)$.

$\begin{array}{lc}\text { Características } & \mathrm{n}(\%) \\ \text { Sexo } & \\ \quad \text { Femenino } & 19(57.58) \\ \quad \text { Masculino } & 14(42.42) \\ \text { Edad (años), mediana (mín.-máx.) } & 52(30-79) \\ \text { Estado funcional por ECOG } & \\ 0 & 19(57.60) \\ 1 & 8(24.20) \\ 2 & 5(15.20) \\ 3 & 1(3.00) \\ \text { Tipo de mieloma } & \\ \text { IgG } & 22(66.70) \\ \text { IgA } & 6(18.20) \\ \text { Cadenas ligeras } & 4(12.10) \\ \text { No secretor } & 1(3.00)\end{array}$

ECOG = Eastern Cooperative Oncology Group; mín.-máx. = mínimomáximo; IgG = inmunoglobulina $\mathrm{G}$; $\lg \mathrm{A}=$ inmunoglobulina $\mathrm{A}$. patológico y la compresión en el conducto medular. Todos estos efectos influyen en el tratamiento de la enfermedad, los requerimientos de cirugía, de radioterapia paliativa, así como en la calidad de vida y pronóstico del paciente. ${ }^{14} \mathrm{Se}$ ha reportado que la presencia de una fractura al momento del diagnóstico o durante la evolución de la enfermedad incrementa el riesgo de muerte. ${ }^{15}$

En el presente estudio encontramos que, de nuestra población total, 9.1\% de los pacientes presentaron una fractura que llevó a la sospecha de mieloma múltiple. En un estudio realizado por Thorsteinsdottir, en Suiza, encontraron que hasta en $8.7 \%$ de los pacientes presentaron una fractura 30 días previos o inmediatamente después del diagnóstico de mieloma múltiple. Sin embargo, 66.6\% de los pacientes presentaban características clínicas de 
la EOMM, lo que nos predice un factor importante que se omite ante el diagnóstico de esta patología, ya que la EOMM pronostica una mayor morbilidad en estos pacientes por un estadio avanzado de la enfermedad.

En nuestro estudio encontramos que las fracturas de cuerpos vertebrales, húmero, cadera y fémur son las más frecuentes, lo que coincide con la publicación de Thorsteinsdottir, en la que se encontró un mayor riesgo de muerte en aquellos pacientes con fracturas en estas localizaciones. Nosotros no realizamos un seguimiento de supervivencia, pero sería importante considerar en posteriores investigaciones si tiene una distribución similar a la descrita en estudios con muestras más grandes.

El método radiológico de evaluación es relevante. En la literatura el más utilizado es la serie ósea metastásica, por lo que es el que utilizamos en nuestro hospital. Sin embargo, la capacidad de detección EOMM en pacientes al debut con este método fue sólo de $9 \%$, aun cuando los pacientes referían sintomatología de la EOMM. Al realizar estudios complementarios a través del tiempo, se documentó la EOMM hasta en cerca del 80\% de los pacientes. Esto obliga a buscar eventos relacionados al esqueleto con estudios de mayor sensibilidad como la tomografía o resonancia magnética o incluso PET SCAN/CT, ya que pueden identificarse lesiones más pequeñas y en etapas previas a fracturas. ${ }^{16}$

La presencia de lesiones líticas mayores de $25 \mathrm{~mm}$, o en zonas de gran carga o áreas de reabsorción mayor de 50$75 \%$ del diámetro óseo, siempre tendrán un riesgo mayor de presentar fractura.

El manejo de estas fracturas debe consistir en su estabilización, sobre todo cuando se asocian con inestabilidad de los huesos largos. Sin embargo, el manejo quirúrgico debe valorarse de forma individual, tomando en cuenta la localización de la fractura, las lesiones asociadas con la misma, el riesgo inminente de fractura, el estado de salud del paciente y el pronóstico al dar un manejo quirúrgico. ${ }^{17}$ Al mismo tiempo, el manejo de la EOMM tiene un abordaje multimodal y puede ser desde la parte preventiva hasta la paliativa.

De los principales tratamientos que se llevan a cabo como métodos preventivos tenemos desde el uso de los bifosfonatos, medicamentos que influyen en la respuesta celular de la enfermedad como el denosumab y el ácido zoledrónico, hasta el uso de radioterapia para manejo paliativo al dolor óseo generalizado.

\section{CONCLUSIÓN}

El 9.1\% de los pacientes de nuestro estudio tuvieron una fractura que presentaba características radiológicas de proceso neoplásico, con sospecha y posterior diagnóstico confirmatorio de mieloma múltiple. Durante el seguimiento se diagnosticaron 24 pacientes con alguna fractura asociada al mieloma múltiple, siendo más frecuentes en columna, húmero y cadera.

Se requiere un seguimiento de supervivencia para valorar el impacto de las fracturas en la calidad de vida y pronóstico de los pacientes.

\section{REFERENCIAS}

1. Gerecke C, Fuhrmann S, Strifler S, Schmidt-Hieber M, Einsele $H$, Knop S. The diagnosis and treatment of multiple myeloma. Dtsch Arztebl Int. 2016; 113 (27-28): 470-476.

2. Siegel R, Naishadham D, Jemal A. Cancer statistics, 2013. CA Cancer J Clin. 2013; 3 (1): 11-30.

3. Jemal A, Siegel R, Ward E, Hao Y, Xu J, Thun MJ. Cancer Statistics, 2009. CA Cancer J Clin. 2009; 59 (4): 225-249.

4. Alvarado-Ibarra M, Álvarez-Vera JL, Anaya-Cuéllar I, De la PeñaCelaya A, García-Fernández L, Hernández-Ruiz E et al. Primer Consenso Nacional de Mieloma Múltiple por Hematólogos del ISSSTE. Rev Hematol Mex. 2015; 16 (4): 306-332.

5. Ries LAG, Young JL, Keel GE, Eisner MP, Lin YD, Horner MJ (editors). SEER survival monograph: cancer survival among adults: U.S. SEER Program, 1988-2001, patient and tumor characteristics. National Cancer Institute, SEER Program. NIH Pub. No. 076215, 2007.

6. Brigle K, Rogers B. Pathobiology and diagnosis of multiple myeloma. Semin Oncol Nurs. 2017; 33 (3): 225-236.

7. Walker RE, Lawson MA, Buckle CH, Snowden JA, Chantry AD. Myeloma bone disease: pathogenesis, current treatments and future targets. Br Med Bull. 2014; 111 (1): 117-138.

8. Terpos E, Christoulas D, Gavriatopoulou M. Biology and treatment of myeloma related bone disease. Metabolism. 2018; 80: 80-90.

9. Melton LJ 3rd, Kyle RA, Achenbach SJ, Oberg AL, Rajkumar SV. Fracture risk with multiple myeloma: a population-based study. J Bone Miner Res. 2005; 20 (3): 487-493.

10. Herget GW, Wasch R, Klein L, Schmal H, Terpos E, Engelhardt $\mathrm{M}$. Prevention of bone disease and early detection of impending fractures in multiple myeloma patients can reduce morbidity and mortality: the necessity of interdisciplinary state-of-the-art treatment. Haematologica. 2020; 105 (4): 859-861.

11. Abildgaard N, Brixen K, Kristensen JE, Vejlgaard T, Charles P, Nielsen JL. Assessment of bone involvement in patients with multiple myeloma using bone densitometry. Eur J Haematol. 1996; 57 (5): 370-376.

12. Rajkumar SV, Dimopoulos MA, Palumbo A, Blade J, Merlini G, Mateos $\mathrm{MV}$ et al. International Myeloma Working Group updated criteria for the diagnosis of multiple myeloma. Lancet Oncol. 2014; 15 (12): 538-548.

13. Sharma AM, Sackett M, Bueddefeld D, Lambert P, Dubey A, Wadhwa $\checkmark$ et al. Incidence of spinal disease and role of spinal radiotherapy in multiple myeloma. Curr Oncol. 2018; 25 (6): 539-544.

14. Terpos E, Morgan G, Dimopoulos MA, Drake MT, Lentzsch S, Raje $\mathrm{N}$ et al. International Myeloma Working Group recommendations for the treatment of multiple myeloma-related bone disease. J Clin Oncol. 2013; 31 (18): 2347-2357.

15. Thorsteinsdottir S, Gislason G, Aspelund T, Sverrisdottir I, Landgren O, Turesson I et al. Fractures and survival in multiple myeloma: Results from population-based study. Haematologica. 2020; 105 (4): 1067-1073.

16. Derlin T, Bannas P. Imaging of multiple myeloma: current concepts. World J Orthop. 2014; 5 (3): 272-282.

17. Bryson DJ, Wicks L, Ashford RU. The investigation and management of suspected malignant pathological fractures: a review for the general orthopaedic surgeon. Injury. 2015; 46 (10): 1891-1899. 